\title{
Il consenso informato in aferesi: il punto di vista del nefrologo
}

\author{
Gaspare Elios Russo, Tania Gnerre Musto, Massimo Testorio, Anna Rita D'Angelo, \\ Silvia Lai, Barbara Borzacca, Augusto Morgia, Monica Serraiocco, Marta Casarci, \\ Alessandra Nunzi, Andrea Martinez
}

U.O.S. Dialisi Peritoneale, D.A.I. Nefrologia-Urologia, Dip. di Scienze Ginecologico-Ostetriche e Scienze Urologiche, Policlinico Umberto I, Sapienza, Università di Roma, Roma

.O.S. Dialisi Peritoneale, D.A.I. Nefrologia-Urologia, Dip. di Scienze Ginecologico-Ostetriche e Scienze Urologiche, Policlinico Umberto I, Sapienza, Università di Roma, Roma

\begin{abstract}
INFORMED CONSENT IN APHERESIS: THE NEPHROLOGIST'S POINT OF VIEW
Abstract. Since its first use apheresis has changed, developing in terms of different materials and techniques, and has been applied to treat several types of diseases. Thus, apheresis has become a complex therapeutical device that requires particular knowledge and expertise.

Overall the use of this technique has been poorly managed in Italy, as well as in Europe, since physicians cannot refer to specific guidelines for its application. The purpose of a study group created in 1993 by the Italian Nephrology Society, is to develop such guidelines and protocols with a multidisciplinary approach. This allows different specializations to bring their knowledge together in a collaboration that will not only result in the realization of a common goal, but will also allow to clarify specific aspects of their own specialty. Informed consent is a fundamental act in medicine, as it legitimates every medical action. We suggest using a standard process to obtain the consent in any case requiring apheresis.
\end{abstract}

Key words: Informed consent, Apheresis, Multidisciplinary approach, Deontological ethics

Conflict of interest: None.

Financial support: None.

Ricevuto: 28 Gennaio 2013; Accettato: 21 Marzo 2013

\section{Introduzione}

Il consenso informato acquisisce un ruolo fondamentale nel rapporto medico-paziente, che è alla base dell'applicazione di qualsiasi trattamento, terapia o tecnica medica.

Anche nell'ambito dell'aferesi terapeutica, l'acquisizione del consenso gioca un ruolo fondamentale.

\section{Considerazioni storiche}

Nel 1500 scriveva il Botallo:

"Come da un pozzo si estrae acqua torbida fino a che dalla sorgente sotterranea non venga acqua pura soltanto, cosi va cavato sangue corrotto fino a che non rimanga altro che quello puro" (1).

Questo concetto può essere visto come il principio base dell'aferesi.

È nel 1913 che avviene la prima pubblicazione sulla dialisi ad opera di Abel, Rowntree e Turner, che l'anno successivo pubblicarono: Plasma removal with return of corpuscles (Plasmaphaeresis) $(2,3)$.

Viene introdotto, così, il termine di "plasmaferesi" per indicare la rimozione dal sangue di sostanze tossiche, così da considerare Abel a tutti gli effetti il padre sia della dialisi che dell'aferesi. Dovranno passare altri 40 anni prima che, nel 1952, venga utilizzata la plasmaferesi su un paziente affetto da mieloma multiplo (4). Le prime vere applicazioni cliniche risalgono al 1963 ad opera di Salomon e Fahey in soggetti affetti da paraproteinemia, per ridurre l'iperviscosità del sangue (5).

L'aferesi ha conosciuto nel tempo, per evoluzione dei materiali d'uso, diversificazione delle tecniche (LDL aferesi, plasmaferesi a cascata, ecc.) e possibilità di impiego clinico, un progresso tale da rappresentare oggi un presidio terapeutico assai complesso che richiede specifiche conoscenze e competenze (6-11). In Italia, come nel resto dell'Europa, questa attività clinica è stata gestita in maniera poco attenta, tanto che, a oggi, non si riconoscono né degli specifici ambiti di impiego né i settori di utenza (12). Per tale motivo, nel 1993, si è costituito, in seno 
alla Società Italiana di Nefrologia, il gruppo di studio dell'aferesi terapeutica che si prefiggeva il compito di censire tutte le strutture in ambito nefrologico che utilizzavano o intendevano utilizzare tecniche aferetiche per scopi terapeutici e di coinvolgere tutti gli operatori in un programma comune per lo sviluppo di un corpus unico di protocolli e Linee Guida di riferimento.

\section{La multidisciplinarietà dell'aferesi}

\begin{abstract}
"In letting of blood three main circumstances are to be considered, Who, how much, when. That is, that it be done to such a one as may endure it, or to whom it may belong, that he be of a competent age, not too young, nor too old, overweak, fat, or lean, sore laboured, but to such as have need, are full of bad blood, noxious humours, and may be eased by it."

$$
\text { Burton: "The Anatomy of Melancholy", } 1640
$$
\end{abstract}

Queste raccomandazioni per l'applicazione del salasso suonano ancora attuali per l'aferesi, ovvero a chi farla, in quale dose e quando cominciare. Data la varietà dei campi di impiego dell'aferesi, vale a dire reumatologia, nefrologia, ematologia e così via, sono diverse le discipline coinvolte che possono dare il loro apporto alla stesura di un testo per l'appropriata gestione della metodica (5). Infatti, in assenza di una specifica o univoca indicazione terapeutica, si rende necessario l'apporto di competenze dei diversi specialisti che gravitano intorno alla materia. Attraverso mezzi quali i registri nazionali e internazionali e la progettazione di trial, si potrà approdare, infine, alla redazione di vere e proprie Linee Guida condivise e a idonei marker che identifichino l'appropriatezza e l'efficacia della terapia.

La multidisciplinarietà rappresenta un concetto moderno di operare per la ricostruzione delle singole discipline, aperte allo scambio e solidali nella ricerca di nuovi percorsi. Prendendo in prestito i metodi e le teorie di un'altra disciplina, spesso è possibile chiarire alcuni problemi di quella in cui si opera e suscitare un dialogo sistematico e produttivo tra le aree in contatto.

\section{Aspetti normativi e medico-legali}

La nostra opinione è che il medico debba riportarsi, nell'applicazione della procedura aferetica, ancora prima che a quelle leggi che ci danno alcuni indirizzi normativi, al Codice di Deontologia Medica, derivato dal Giuramento di Ippocrate, e, nel campo della bioetica, al principio di autonomia secondo Kant (Beneficentia, non maleficentia, Autonomia).

Il codice deontologico è un corpus di regole di autodisciplina vincolanti per l'esercizio della professione. Nel codice troviamo il riconoscimento della potestà () del medico per quanto riguarda tutti quei casi in cui la "medicina ufficiale" non dia delle indicazioni chiare.

In questi, pertanto, il medico può ricorrere, agendo secondo scienza e coscienza, a terapie o a metodi allo stato non compresi nell'"ufficialità", quando egli reputi che questi possano risultare utili per il paziente.

Il principio di autonomia è fondato sull'idea che le azioni umane non dovrebbero essere sottoposte ad alcun vincolo o controllo altrui: nel nostro caso, si traduce nel diritto di non interferenza (privacy, riservatezza) e nel riconoscimento del ruolo dell'autodeterminazione, in senso positivo, che implica il dovere di informare e, quindi, di rendere possibili scelte realmente autonome, rispettando tre criteri:

1. la mancanza di condizionamenti esterni;

2. l'intenzionalità;

3. la comprensione della situazione.

La disciplina dell'aferesi trova pochi riferimenti legislativi che qui riportiamo.

La legge 107/90 si esprime sull'organizzazione del lavoro dell'attività trasfusionale e, quindi, sulla divisione dei compiti e delle responsabilità. A tale riguardo va ricordato che al responsabile del servizio spetta il compito di dare indicazioni su procedimenti, protocolli e Linee Guida, nonché sulla scelta e sull'uso delle apparecchiature, dei presidi e così via.

Infatti, l'Art. 9 del D.M. 27/12/1990 indica il personale laureato in medicina e chirurgia come competente per:

- la diagnosi, la cura e l'appropriatezza della prestazione;

- l'indicazione delle tecniche più idonee nella circostanza;

- la supervisione dell'operato dei collaboratori con minore qualificazione professionale (infermieri, studenti, specializzandi, ecc.).

Già una sentenza della Corte di Cassazione n. 7082 del 27/07/1989 si esprimeva in tal senso: "L'attività ausiliaria o di assistenza al malato deve essere svolta sempre sotto il controllo medico".

Ne deriva, pertanto, che, ogni volta che un danno causato al paziente possa essere imputato, in tutto o in parte, all'organizzazione del lavoro, questo comporterà il coinvolgimento del responsabile del servizio nei seguenti casi:

- per aver dato delle direttive non idonee;

- per non aver dato le opportune direttive;

- per non aver supervisionato l'operato dei propri collaboratori.

Il consenso informato si inserisce in questo discorso, in quanto è l'espressione della volontà del cittadino malato, che autorizza il medico a effettuare uno specifico trattamento medicochirurgico sulla sua persona. Non si può prescindere da tale consenso, fatte salve le condizioni previste dalla legge (urgenza, incapacità di intendere e di volere), perché qualunque azione effettuata senza tale autorizzazione sarebbe illecita da un punto di vista penale.

Occorre avere la consapevolezza che il medico non ha il "diritto di curare", ma solo la "potestà" e la "facoltà" di curare su richiesta del paziente, al di fuori dei casi di emergenza. Per questo, il consenso del paziente alle cure, indipendentemente dai risultati che si potranno ottenere, non è solamente un obbligo deontologico e contrattuale, quanto, piuttosto, l'atto primario che legittima il trattamento medico-chirurgico per ogni singolo paziente.

La violazione all'obbligo del consenso informato determina per il medico una responsabilità sia disciplinare che penale e civile a norma degli articoli 2, 13 e 32 della Costituzione e degli articoli 32-38 del Codice di Deontologia Medica. Riassumendo, per essere valido, il consenso deve rispettare $\mathrm{i}$ seguenti requisiti: 


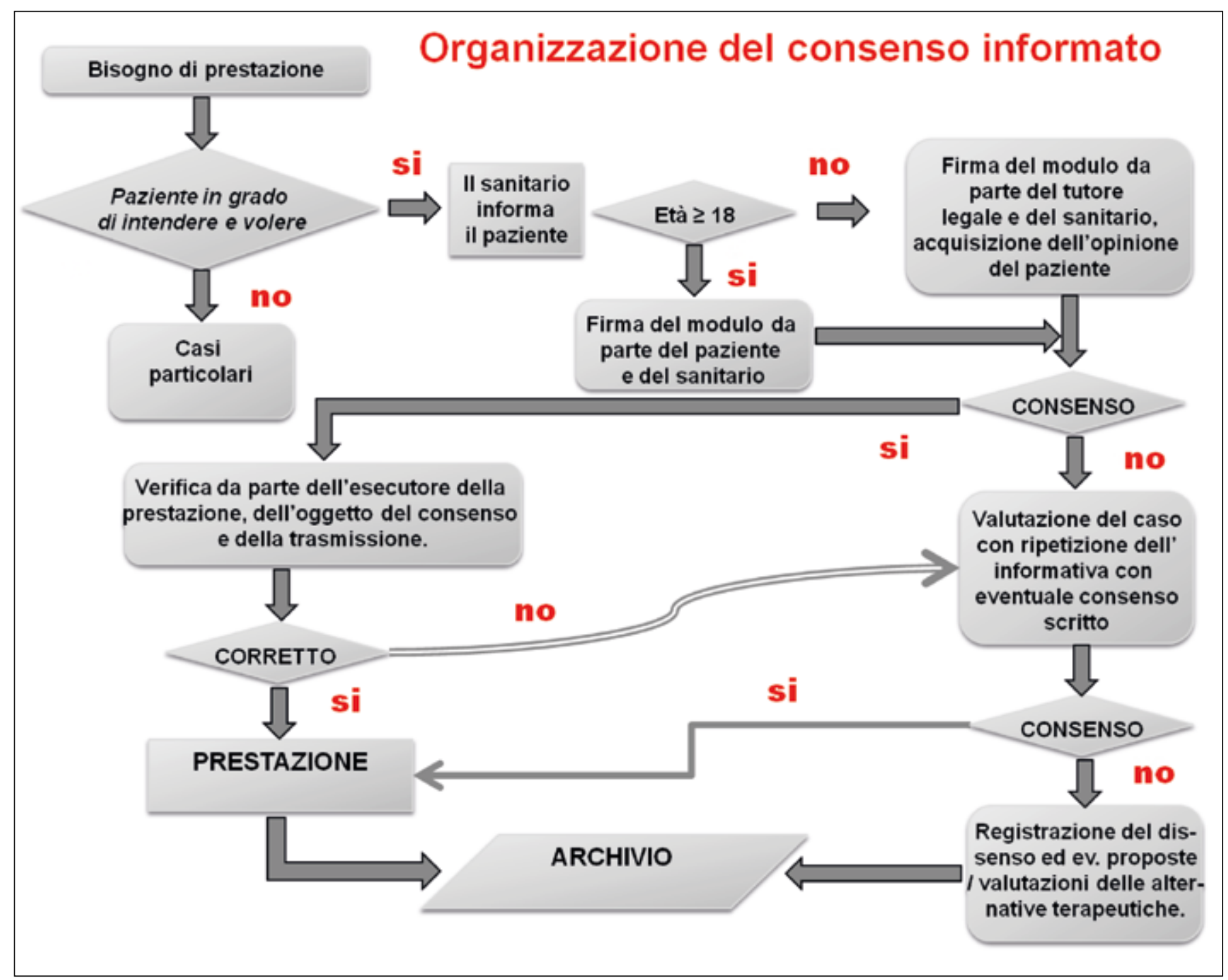

Fig. 1 - Procedura di acquisizione del consenso informato.

- deve essere espresso da un individuo capace di intendere e di volere;

- va fornita un'informazione esauriente: notizie chiare sulla patologia del soggetto e su modalità e rischi che il trattamento può comportare;

- informare su eventuali interventi o terapie alternative;

- deve essere libero, frutto di un convincimento personale del paziente che ha già ricevuto tutte le informazioni necessarie;

- può essere revocato in qualsiasi momento, anche poco prima del trattamento.

Di seguito riportiamo un diagramma di flusso (Fig. 1), che indica come potrebbe essere svolta la procedura per l'acquisizione del consenso rispettando le precedenti raccomandazioni.
Tenuto conto dell'ampiezza del campo di applicazione della disciplina aferetica e della carenza legislativa in merito, proponiamo un modulo-tipo da usare come base per informare il paziente e per acquisire il consenso al trattamento, presto reperibile sul sito del Gruppo di Studio della SIN.

\section{Riassunto}

L'aferesi ha conosciuto nel tempo, per evoluzione dei materiali d'uso, diversificazione delle tecniche e possibilità di impiego clinico, un progresso tale da rappresentare un presidio terapeutico complesso che richiede specifiche conoscenze e competenze.

In Europa, questa attività clinica è stata gestita in maniera poco attenta, tanto che, a oggi, non si riconoscono specifici 
ambiti di impiego né settori di utenza. Per tale motivo, nel 1993, si è costituito, in seno alla Società Italiana di Nefrologia, il Gruppo di Studio dell'aferesi terapeutica.

Infatti, in assenza di una specifica o univoca indicazione terapeutica, si rende necessario l'approccio multidisciplinare, che dovrà approdare alla redazione di Linee Guida condivise e a idonei marker di appropriatezza e di efficacia della terapia.

Il consenso informato, espressione della volontà del cittadino malato che autorizza il medico a compiere uno specifico trattamento medico-chirurgico, acquisisce un ruolo fondamentale anche nell'ambito dell'aferesi terapeutica. Il medico non ha il "diritto di curare", ma solo la "potestà" e la "facoltà" di curare su richiesta del paziente, al di fuori dei casi di emergenza. Pertanto, il consenso non è solamente un obbligo deontologico-contrattuale ma l'atto che legittima il trattamento per ogni singolo paziente.

Quindi, suggeriamo un percorso standard per l'acquisizione del consenso nell'ambito dell'aferesi.
Parole chiave: Consenso informato, Aferesi, Approccio multidisciplinare, Deontologia

Dichiarazione di conflitto di interessi: Gli Autori dichiarano di non avere conflitto di interessi.

Contributi economici degli autori: Gli Autori dichiarano di non aver ricevuto sponsorizzazioni economiche per la preparazione dell'articolo.

Indirizzo degli Autori:

Prof. Gaspare Elios Russo

D.A.I. Nefrologia-Urologia

Dialisi Peritoneale, $3^{\circ}$ Piano

Policlinico Umberto I

Viale del Policlinico 155

00161 Roma

gasparelios.russo@uniroma1.it

\section{Bibliografia}

1. Botallo L. De incidendae venae, cutis scarificandae et Hirudinum applicandarum modo. Lugduni 1565.

2. Abel JJ, Rowntree LG, Turner BB. Plasma removal with return of corpuscles (plasmaphaeresis). The Journal of Pharmacology and experimental therapeutics Vol. V. No. 6, July, 1914. Transfus Sci 1990; 11 (2): 166-77.

3. Abel JJ, Rowntree LG, Turner BB. On the removal of diffusable substances from the circulating blood by means of dialysis. Transactions of the Association of American Physicians, 1913. Transfus Sci 1990; 11 (2): 164-5.

4. Adams WS, Blahd WH, Bassett SH. A Method of Human Plasmapheresis. Proc Soc Exp Biol Med 1952; 80: 377-9.

5. Solomon A, Fahey JL. Plasmapheresis therapy in macroglobulinemia. Ann Intern Med 1963; 58: 789-800.

6. Russo GE, Cavallini M, Centi A, Baliva G, Carraro M, Pugliese F. Extracorporeal photochemotherapy for the treatment of glomerulopathies with associated nephrotic syndrome. J
Nephrol 2010; 23 (1): 85-9.

7. Russo GE, Bonello M, Vitaliano E, et al. Plasmapheresis combined with pharmacology in the treatment of lupus nephritis: a new therapeutic protocol. Clin Ter 2000; 151 (1): 9-13.

8. Russo GE, Bonello M, Bauco B, et al. Nephrotic syndrome and plasmapheresis. Int J Artif Organs 2000; 23 (2): 111-3.

9. Russo GE, Giusti S, Maurici M, et al. [Plasmapheresis and mushroom poisoning: report of a case of Amanita phalloides poisoning]. Clin Ter 1997; 148 (5-6): 277-80.

10. Russo GE, Caramiello MS, Vitaliano E, et al. Haemorheological changes in mixed cryoglobulinaemia during apheresis treatment. Transfus Sci 1996; 17 (4): 499-503.

11. Russo GE, Fagiolo M, Vitaliano E, Caramiello MS. Biocompatibility in hemapheresis: new materials. Int J Artif Organs 1993; 16 (Suppl. 5): 214-6.

12. De Silvestro G, Marson P, Russo GE, Vicarioto M, Donadel C. National survey of apheresis activity in Italy (2000). Transfus Apher Sci 2004; 30 (1): 61-71. 